\title{
The experimental evaluation of the EGNOS safety-of-life services for railway signalling
}

\author{
A. Filip, L. Bažant \& H. Mocek \\ Railway Infrastructure Administration, LIS, Pardubice, Czech Republic
}

\begin{abstract}
This article describes the theoretical and experimental investigation of the satellite navigation based Safety-of-Life (SoL) services intended for railway safety-related applications. Main attention is paid to the EGNOS Precision Approach (PA) and Non-precision Approach (NPA) navigation modes, which were mainly designed according to the specific aeronautical requirements.

Two basic research approaches have been used: 1) a recently developed methodology for the description of the GNSS quality measures in terms of railway dependability attributes, and 2) an operational EGNOS system with SoL receivers. The practical results include the theoretical analysis of the EGNOS SoL services for railway signalling and the experimental evaluation of the EGNOS PA and NPA operational modes in the laboratory and trial area.
\end{abstract}

Keywords: GPS, EGNOS, RAMS, RAIM, SBAS, Galileo Safety-of-Life Service, integrity risk, satellite navigation, railway safety, signalling.

\section{Introduction}

Global Navigation Satellite Systems (GNSS), such as the US Wide Area Augmentation System (WAAS) [1], the European Geostationary Navigation Overlay System (EGNOS) [1], the Japanese MSAT and the future Galileo with its Safety-of-Life services [2], have been mainly designed according to the International Civil Aviation Organisation (ICAO) safety requirements. In order to use this promising technology in the railway domain and develop new GNSS based safety-related applications, the dependability attributes of real GNSS SoL services in accordance with the CENELEC railway safety standards are needed.

Generally, it has been assumed that railway signalling could utilise SoL services, such as the Galileo Level A or EGNOS Precision Approach mode. 
These services are dedicated to the precision approach of airplanes, where the integrity risk (probability of dangerous undetected failure) is scaled by the time interval of $150 \mathrm{~s}$. In the case of EGNOS, a great deal of the integrity risk is allocated to the vertical position, since it is critical for airplanes. However, for railways the mainly interest is in determination of the dangerous undetected failure rate (per 1 hour) of the computed horizontal position and other dependability attributes.

Therefore, our attention in this paper is mainly focused on investigation of SoL services originally intended for aeronautical operations, ranging from the En-route to Non-precision Approach, where the integrity risk is allocated to the horizontal position and is scaled by 1 hour. The presented experimental results describe the real behaviour of the EGNOS PA and NPA modes in static and dynamic conditions.

\section{Rationale for the SBAS PA and NPA mode specifications}

When a railway user starts to use for the first time a so called Satellite Based Augmentation System (SBAS) receiver, such as WAAS, MSAT or EGNOS SoL, which is compliant with the standard DO-229D [1], he/she has to decide which of the two navigation modes to select: the PA or the NPA mode. The problem is that none of the navigational modes was designed for railway safety-related applications. In the following paragraphs rationale and background for derivation of the navigational mode integrity requirements are outlined and the main differences between the PA and NPA modes are described.

\subsection{Protection levels calculation and the integrity risk allocation}

The main differentiator between the standalone GPS and SBAS system is that the SBAS receiver provides to a user the vertical and horizontal protection levels (VPL and HPL) for each computed position, e.g. every 1 second. The protection levels (in meters) bound the provided position with the given integrity risk. In the case of the PA mode the acceptable Signal-In-Space integrity risk is maximally $2 \times 10^{-7} / 150 \mathrm{~s}$ and in the case of NPA the total integrity risk should not exceed $1 \times 10^{-7} / 1$ hour. The calculated values of the protection levels are proportional to the maximal estimated standard deviation $\sigma_{\max }$ of the error model and the integrity requirement is implemented to the protection level by the scaling coefficient $K$. The coefficient $K$ is derived from the cumulative probability distribution function (e.g. Gaussian, Rayleigh) corresponding to the specific aeronautical operational requirements. For example, the HPL equation [1] can be written as follows

$$
H P L=K_{H} \cdot \sigma_{H, \max }=K_{H} \cdot f(\text { satellite geometry, residual errors of diff. corrections })
$$

\subsubsection{PA mode}

In Figs. 1 and 2 there are outlined the integrity risk allocation trees for the SBAS PA and NPA modes, respectively, which result from aeronautical requirements. In the case of the PA mode, the total integrity risk of $2 \times 10^{-7} / 150 \mathrm{~s}$ is equally 




Figure 1: Integrity risk allocation tree for the precision approach.

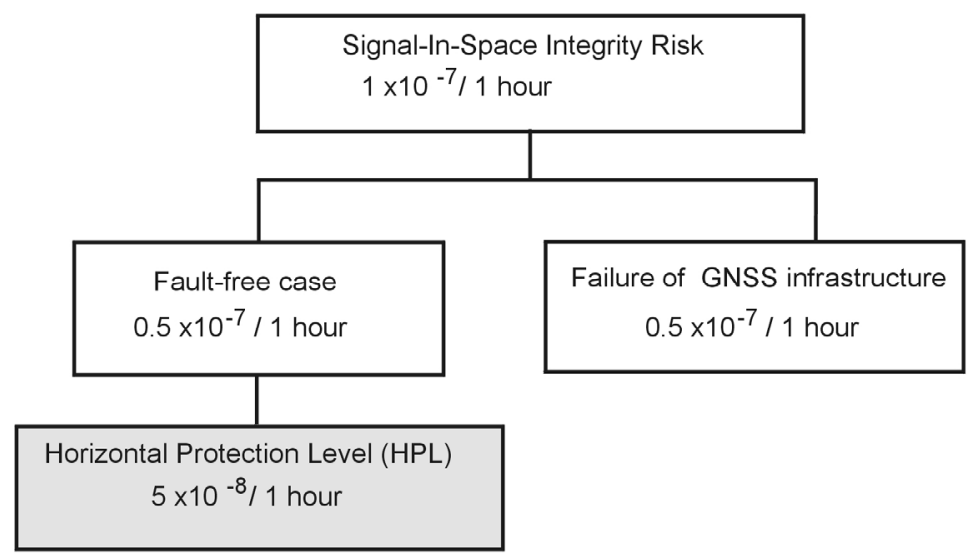

Figure 2: Integrity risk allocation tree for the non-precision approach.

divided between the integrity risk of the system (EGNOS ground segment, satellites...) and the so called fault-free integrity risk, which is induced by the measurement noise of the ground segment and algorithmic processing.

As is evident from Fig. 1, most of the fault-free integrity risk is allocated to the vertical position and only a minimal value of the risk $\left(2 \times 10^{-9} / 150 \mathrm{~s}\right)$ is allocated to the horizontal position. The position guarantee in the vertical direction is much more important for the PA mode. Further, the guarantee of position in the horizontal lateral direction only (i.e. one-dimensional) is required for the precision approach with vertical guidance. No two-dimensional guarantee in the horizontal plane is needed. Finally, aviation requires a position guarantee 
for PA operations within the time interval of $150 \mathrm{~s}$. Only one independent sample (measurement) is needed during this exposure interval. What is behind the interval of $150 \mathrm{~s}$ is not important for the PA mode. Due to the above reasons, the PA requirements are not applicable for railway safety-related applications.

\subsubsection{NPA mode}

In the case of the aeronautical requirements for the NPA mode, the entire SIS integrity risk of $1 \times 10^{-7} / 1$ hour is allocated to the position in the horizontal plane. In this case, the time scale of 1 hour is also acceptable for land applications. As is evident from Fig. 2, the total integrity risk is equally allocated between the fault-free case $\left(0.5 \times 10^{-7} / 1\right.$ hour $)$ and faulty case $\left(0.5 \times 10^{-7} / 1\right.$ hour $)$. Then the coefficient $K_{H}$ in the HPL equation (1) can be derived as follows. It is assumed, that 10 independent samples exist per 1 hour. Thus, the fault-free integrity risk per one independent sample is $0.5 \times 10^{-8} / 1$ hour. Since the position determination requirement for the NPA mode is a two-dimensional problem, then the $K_{H, N P A}$ coefficient can be derived from the Rayleigh probability distribution as

$$
K_{H, N P A}=\text { Rayleigh } c d f^{-1}\left(1-P_{m d}\right)=\text { Rayleigh } c d f^{-1}\left(1-5 \times 10^{-9}\right)=6.18
$$

where $P_{m d}$ means the probability of missed detection. It seems that the SBAS NPA mode much more meets railway needs than the PA mode. However, to be really sure that the NPA mode could be applied for railway safety-related applications, it is necessary not only to determine the error decorrelation time (number of independent measurements) in a real SBAS system, but also to check the algorithms for the calculation of the position and the protection levels. All of these topics will be briefly discussed in the following paragraphs.

\subsection{Influence of decorrelation time on protection level and failure rate}

Recently, a conversion of the GNSS integrity and continuity risks from the time basis of $150 \mathrm{~s}(15 \mathrm{~s})$ to 1 hour scale has been done $[3,4]$. In the case of the Galileo SoL service - Level A and its integrity risk, it was assumed that the time between independent samples exceeds $150 \mathrm{~s}$. Thus, the cumulative probability principle was employed for the failure rate estimation on a 1 hour basis.

In case of SBAS (see section2.1.2), it is assumed that the decorrelation time of SBAS errors is $360 \mathrm{~s}$ (10 independent samples per 1 hour). However, as results from our recent experiments and preliminary conclusions, the decorrelation time of SBAS errors is several times higher than the decorrelation time used for derivation of the $K_{H, N P A}$ coefficient. It is clear that the decorrelation time value influences determination of the protection levels (xPL) and failure rate of the SBAS system. Therefore, this topic will be investigated in detail in near future.

Railway standards require specification of the SBAS dangerous undetected failure rate per 1 hour. With respect to the strong correlation of the measurement errors in the SBAS system, the SIS integrity risk per $150 \mathrm{~s}$ is not simply convertible to the failure rate per 1 hour. These conversions should be done by 
means of experiments and subsequent probabilistic evaluation. The operational EGNOS system with SBAS SoL receivers can be used for this purpose.

\subsection{Differences between SBAS PA and NPA receiver modes}

The estimation of xPLs by the SBAS SoL receiver depends on selection of the navigation mode. In the following paragraphs the differences between the PA and NPA modes implemented in the SBAS receiver are described.

\subsubsection{PA mode}

The PA navigation mode is enabled when the position fix computation meets the DO-229D [1] criteria for the precision approach: 1) all satellites used for ranging are SBAS healthy, 2) all satellites are not GPS unhealthy due to a failure of parity or due to default navigation data, 3) all satellites used for ranging have UDREI (i.e. User Differential Range Error Indicator) < 12,4) all satellites used for ranging have an elevation angle above 5 degrees, 5) SBAS fast corrections are applied to all satellites used for ranging, 6) SBAS long-term corrections are applied to all GPS satellites used for ranging, 7) SBAS range-rate corrections are applied to all satellites used for ranging, 8) all satellites used for ranging have SBAS ionospheric corrections applied, 9) all used SBAS integrity and correction data was obtained from a single SBAS GEO, and 10) at least 4 satellites meeting the above conditions are available for position fix computation.

\subsubsection{NPA mode}

The NPA mode is still enabled though the following PA mode conditions (see 2.3.1) are not fulfilled: 1) ionospheric corrections are not all the time required, and 2) it is not mandatory to receive the integrity and correction data from the same SBAS GEO. When the SBAS ionospheric corrections are not available, the standard GPS ionospheric model will be used. In the NPA mode, longer degradation (time-outs) for fast corrections is also allowed. Both the absence of the SBAS ionospheric corrections and the longer fast corrections time-outs can cause larger error in the horizontal position (HPE) and in HPL calculation. However, as it will be experimentally demonstrated in the next paragraph, such HPE and HPL degradations haven't been observed. Even if this case would happen, these undesirable errors can be detected by means of a build-in diagnostic and compensated by means of additional sensors based on physically diverse principles. In spite of less demanding aeronautical requirements for NPA mode, this navigation mode seems more acceptable for railway safety-related applications than the PA mode.

\section{Experimental results: PA versus NPA modes}

An example of the measured data by the SBAS receiver PolaRx3 (Septentrio) in the NPA mode with known position of its antenna is illustrated in Fig. 3. The data was recorded every 1 second in the period from Sept. 19 to Sept. 22, 2009 in our laboratory. The length of the record is $3 \times 10^{5} \mathrm{~s}$ ( $\sim 3.5$ day). As it is evident from the graph in Fig. 3(a), the magnitude of the horizontal protection level 
(HPL) is usually in the range from 10 to $12 \mathrm{~m}$, but it also several times reaches values above $15 \mathrm{~m}$. The absolute horizontal position error (HPE) outlined in Fig. 3(d) doesn't exceed values of $2 \mathrm{~m}$ or 3 meters. Both HPL and HPE depend on a number of the GPS satellites used in the position calculation (Fig. 3(b)) and on the geometric factor HDOP (Fig. 3(c)). However, as results from the additional measurements (not presented in this paper), HPL can also very

(a)

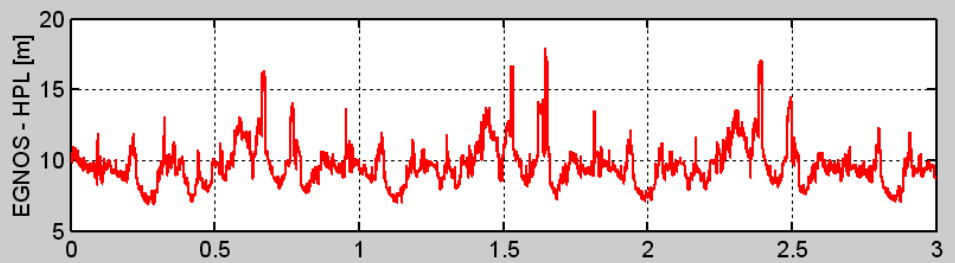

(b)

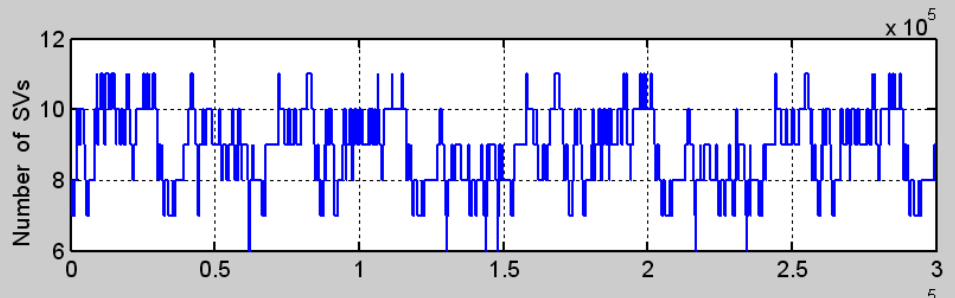

(c)
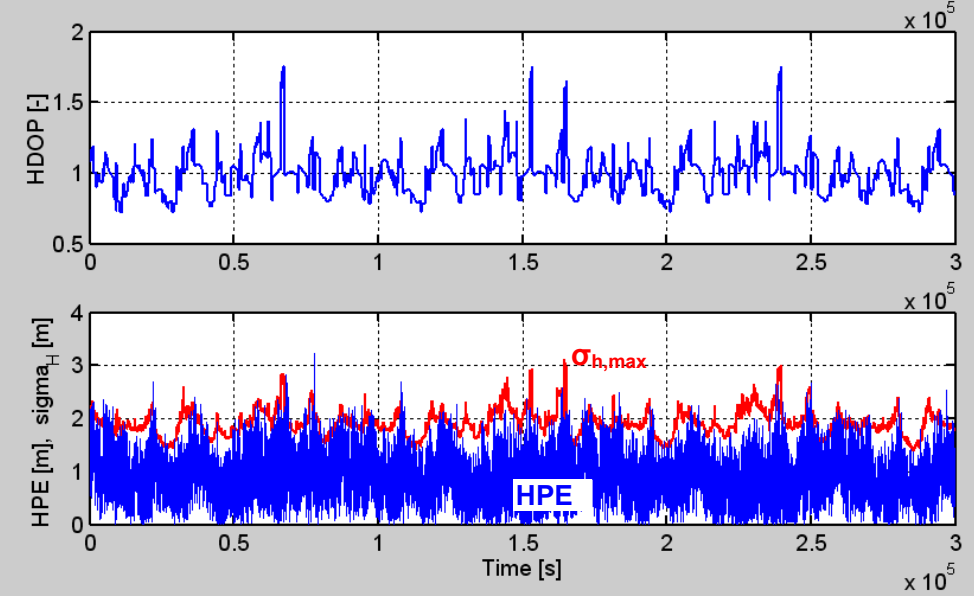

Figure 3: The static measurements by means of the PolaRx3 receiver in the NPA mode: (a) the horizontal protection level (HPL), (b) number of GPS satellites, (c) the horizontal dilution of precision (HDOP), (d) the horizontal position error (HPE), and the estimated standard deviation $\sigma_{\mathrm{H}}$ of the horizontal position error - i.e. the major semiaxis of the error ellipse. 
(a)

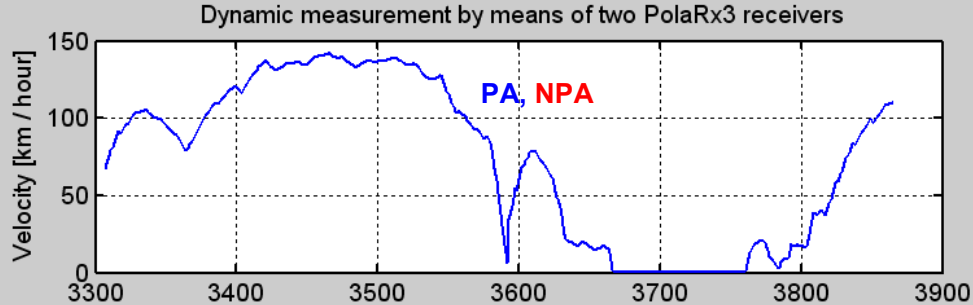

(b)

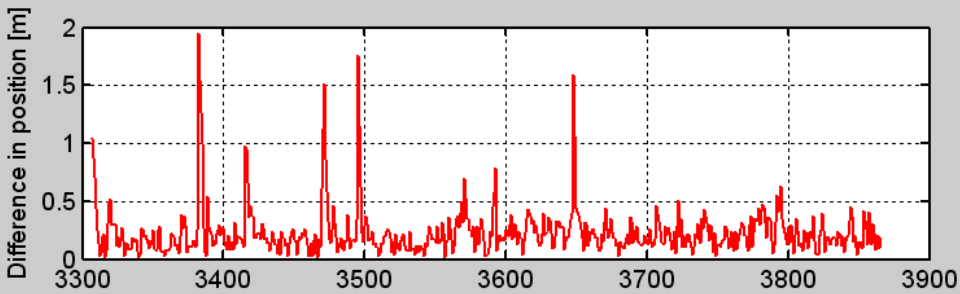

(c)

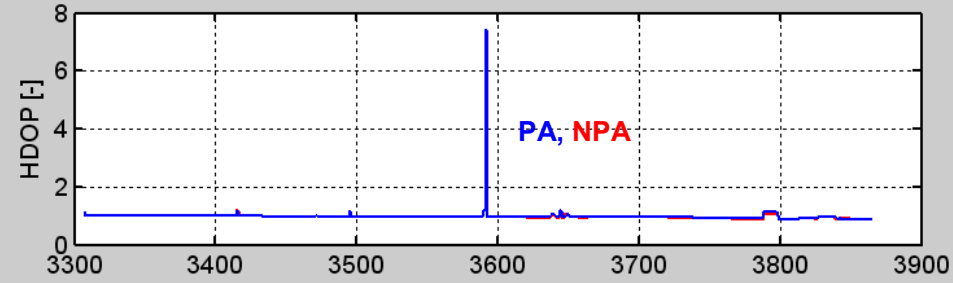

(d)



Figure 4: Dynamic measurements performed by means of two PolaRx3 receivers: (a) the velocity of movement (two overlapped curves), (b) the difference between the corresponding positions provided by two PolaRx3 receivers set up in different navigation modes - PA and NPA, (c) horizontal dilution of precision (HDOP), (d) the number of GPS satellites used in position calculation in the PolaRx3_1 and PolaRx3_2 receivers. 
occasionally, usually for the time interval of a few or tenths of seconds, achieve magnitudes of $60 \sim 100 \mathrm{~m}$ or more. These short-term outliers are currently under investigation. The results of the dynamics measurements performed by using a car with a velocity up to $140 \mathrm{~km} /$ hour are depicted in Fig. 4. The test was performed by means of two identical PolaRx3 receivers. The first PolaRx3 receiver was adjusted in the PA mode, and the other one in the NPA mode. In Fig. 4(a) there is depicted the time dependence of the measured velocity (two identical curves in one). The difference between two corresponding positions provided by both PolaRx 3 receivers is shown in Fig. 4(b). It is evident that the difference between the corresponding positions calculated by the PolaRx3 receivers in diverse navigation modes (PA and NPA) usually doesn't exceed value of $0.5 \mathrm{~m}$. The maximum value of the difference is $2 \mathrm{~m}$. This result is acceptable for railway applications.

\section{RAIM for railway safety-related applications?}

The Receiver Autonomous Integrity Monitor (RAIM) is based on processing of the redundant GPS signals. This technique is usually mentioned as a protection against local effects (multipath, EMI, etc.) that EGNOS is not able to detect. Under normal conditions, when signal from five GPS satellites is received, RAIM is able to detect a failure in the computed fix. When signal from 6 or more satellites is received, then RAIM is able to identify a failure (faulty GPS signal). As it is evident from the diagram in Fig. 5, the horizontal external reliability level (HERL) values provided by the RAIM algorithm are usually larger than the horizontal protection level (HPL) calculated by means of the EGNOS data. The HERL values strongly depend on HDOP - see the corresponding HDOP values

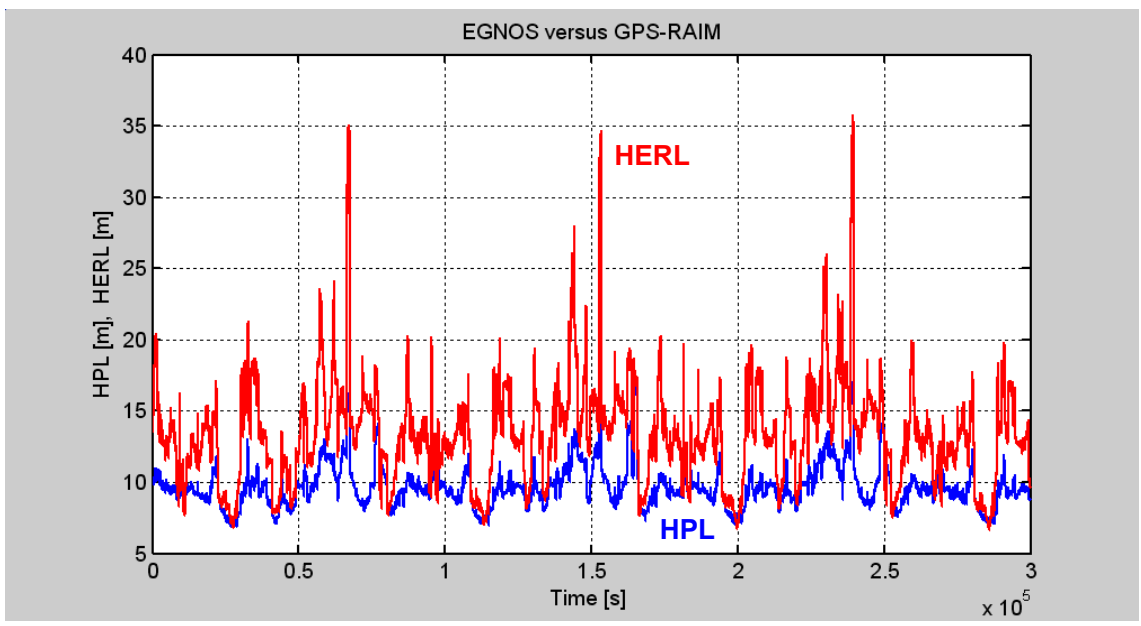

Figure 5: Horizontal protection level (HPL) provided by EGNOS versus horizontal external reliability level (HERL), calculated by the GPS RAIM algorithm in the PolaRx3 receiver. 
in Fig. 3(c). Finally, RAIM doesn't work when less than 5 satellites are used in the position calculation. The RAIM based on a single constellation navigation system is not efficient for railway applications.

\section{Determination of EGNOS dependability attributes}

The aeronautical requirements for the SBAS PA and NPA services [1] cannot be directly used for design and development of railway safety-related applications GNSS based since they do not reflect railway needs. These aeronautical requirements are written in a different language coming from the safety philosophy that differs from the railway one. The already available translated SBAS quality measures into the railway RAM attributes [3, 4] can be only used for a rough estimation of the SBAS application potential to railway environment. These translated RAM attributes are not fully applicable for design, validation and certification of a land safety-related system.

However, the operational EGNOS system exists and it can be used for its dependability determination on an experimental basis in attributes (i.e. failure modes and the corresponding failure rates, reliability and availability corresponding to Alert Limits) conformable with the standard EN 50126 (RAMS) - see Fig. 6.

The initial activities concerning the EGNOS dependability determination for land safety-related applications have been already started in the SŽDC

EGNOS - supports 2 kinds of operations

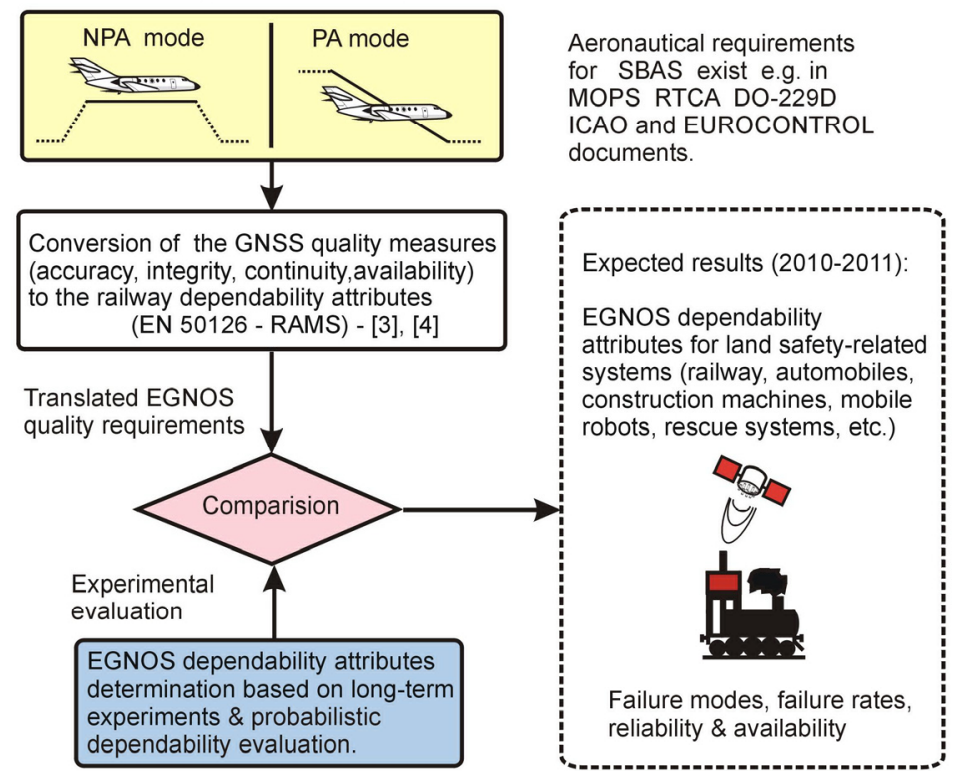

Figure 6: Determination of EGNOS dependability for land applications. 
Laboratory of Intelligent Systems. They have been mainly focused on acquisition and validation of the relevant data. Data from the redundant SBAS receivers are recorded (two PolaRx3 and GG-12W receivers) and there are also processed by the PEGASUS software receiver (EUROCONTROL) in order to avoid possible failures in the user segment. The checked data are evaluated by means of the relevant probabilistic and signal processing methods in order to determine the EGNOS dependability attributes according to the EN 50126 standard.

\section{Conclusion}

In this paper, the EGNOS Non-precision and Precision Approach modes (NPA and PA) for intended railway safety-related applications have been analysed. This investigation was supported by long-term static and dynamic measurements by means of the EGNOS Safety-of-Life receivers. In spite of the fact that none of the above navigation modes was designed according to railway needs, it has been found that the EGNOS NPA navigation mode seems more feasible for land safety-related applications than the PA mode because of the NPA's horizontal integrity risk allocation (not vertical as in the PA mode) and the horizontal integrity risk definition on 1 hour basis (not on $150 \mathrm{~s}$ basis as in the PA mode). However, the final statement whether the NPA mode is really applicable for the railway safety-related applications and how EGNOS should be exactly implemented into safety-related systems will result from the detailed long-term experimental NPA mode observation and from its statistical and probabilistic evaluation. The determination of the EGNOS dependability attributes in terms of failure modes, failure rates (on 1 hour basis), reliability and availability is needed for design, validation and certification of the land GNSS based safety-related systems. It is the current research interest of the authors.

\section{Acknowledgement}

The work was supported by the Ministry of Transport of Czech Republic under contract no. CG743-037-520.

\section{References}

[1] RTCA DO-229D - Minimum operational performance standards for GPS WAAS Airborne Equipment. RTCA, Inc., Washington, D.C., 2006.

[2] Galileo Integrity Concept, ESA document no. ESA-DEUI-NG-TN/01331, (2005).

[3] Filip, A., Beugin, J., Marais, J. and Mocek, H.: Interpretation of the Galileo Safety-of-Life Service by Means of Railway RAMS Terminology. Transactions on Transport Sciences, vol. 1, no. 2, 2008, pp. 61-68.

[4] Filip, A., Beugin, J., Marais, J.: Safety Concept of Railway Signalling Based on Galileo Safety-of-Life Service. COMPRAIL, Toledo, Spain, Sept 15-17, 2008, pp. 103-112. 
[5] Filip, A.: Safety Integrity and Dependability of the EGNOS and Galileo systems. $4^{\text {th }}$ conference - Signalling and Telecommunication Systems on Railway, SŽDC, České Budějovice, Nov 10-12, 2009, pp. 62-65 (in Czech). 